\title{
Assembly of HE800 exopolysaccharide produced by a deep- sea hydrothermal bacterium into microgels for protein delivery applications
}

\author{
Zykwinska Agata ${ }^{1,{ }^{*}}$, Marquis Mélanie ${ }^{2}$, Sinquin Corinne ${ }^{1}$, Cuenot Stéphane ${ }^{3}$, Colliec-Jouault Sylvia ${ }^{1}$ \\ ${ }^{1}$ Ifremer, Laboratoire Ecosystèmes Microbiens et Molécules Marines pour les Biotechnologies, 44311 \\ Nantes, France \\ ${ }^{2}$ INRA, UR1268 Biopolymères Interactions Assemblages, F-44300 Nantes, France \\ ${ }^{3}$ Institut des Matériaux Jean Rouxel (IMN), Université de Nantes-CNRS, 44322 Nantes, France \\ *Corresponding author : Agata Zykwinska, email address : Agata.Zykwinska@ifremer.fr
}

\begin{abstract}
:
Assembly of biopolymers into microgels is an elegant strategy for bioencapsulation with various potential biomedical applications. Such biocompatible and biodegradable microassemblies are developed not only to protect the encapsulated molecule but also to ensure its sustained local delivery. The present study describes the fabrication of microassemblies from a marine HE800 exopolysaccharide (EPS), which displays a glycosaminoglycan (GAG)-like structure and biological properties. HE800 EPS was assembled, through physical cross-linking with divalent ions, into microgel particles and microfibers using microfluidics. The microparticle morphology was highly affected by the polysaccharide concentration and its molecular weight. A model protein, namely Bovine Serum Albumin (BSA) was subsequently encapsulated within HE800 microparticles in one-step process using microfluidics. The protein release was tuned by the microparticle morphology with a lower protein amount released from the most homogeneous structures. Our findings demonstrate the high potential of HE800 EPS based microassemblies as innovative protein microcarriers for further biomedical applications.
\end{abstract}

\section{Highlights}

HE800 marine exopolysaccharide was physically cross-linked to form microgels. Microgel particles and microfibers were structured using microfluidics. Model protein was successfully encapsulated within the microparticles. Microfluidic experiments were supported by numerical simulations.

Keywords : Marine exopolysaccharide, Microgel particles, Microfibers, Microfluidic, Microencapsulation, Protein release 


\section{Introduction}

Hydrogels are biomaterials widely explored for their broad range of applications in medicine and pharmaceutics. These highly hydrophilic three-dimensional polymer networks present some similarities to the extracellular matrix of connective tissue and are therefore frequently used as scaffolds to engineer new tissues in combination with cells and biological signaling molecules (e.g. proteins) (Lee \& Mooney, 2001). Because these biological molecules crucial for cellular responses are extremely fragile, new strategies for their protection and delivery have been developed based on hydrogels structured at micrometer scale (DeFail, Chu, Izzo, \& Marra, 2006; Park, Na, Woo, Yang, \& Park, 2009; Bian et al., 2011; Ansboro et al., 2014). Indeed, microencapsulation of bioactive species allows not only to protect them from external degradation conditions (e.g. chemical, physical or enzymatic), but also offers the possibility for their sustained local delivery, which considerably enhances their bioavailability and efficiency. Hydrogels engineered for biological applications need to fulfill several requirements to ensure their therapeutic efficacy. Because biocompatibility and biodegrability are the most critical parameters, natural polymers such as polysaccharides appear as ideal candidates for the development of smart delivery microgel-based systems. Among all polysaccharides, alginate (Bian et al., 2011; Silva, Ribeiro, Ferreira, \& Veiga, 2006; Jay \& Saltzman, 2009) and chitosan (Bugamelli, Raggi, Orienti, \& Zecchi, 1998; Niu, Feng, Wang, Guo, \& Zheng, 2009; Koppolu et al., 2014) microgels (e.g. microparticles, microcapsules) have been used widely for controlled delivery of proteins. Due to its polyanionic nature, alginate gels easily through ionic cross-linking in the presence of divalent cations. Mild gelation conditions and the use of non-toxic reactants are extremely important when encapsulating proteins. However, alginate is biologically inert and chemical modifications are required to enhance its bioactivity (Genes, Rowley, Mooney, \& Bonassar, 2004; Freeman, Kedem, \& Cohen, 2008). On the contrary to alginate, chitosan presents some structural similarities with glycosaminoglycans (GAGs), natural constituents of the extracellular matrix, which participate in many biological processes through 
72

73

74

75

76

77

78

79

80

81

82

83

84

85

86

87

88

89

90

91

92

93

94

95

96

specific interactions with growth factors, receptors and adhesion proteins (Casu \& Lindahl, 2001). It

can then be thought that the analogous structure may also have related bioactivities. However, GAGs are all anionic polysaccharides, on the contrary to chitosan, which is a cationic polyelectrolyte. Therefore, chitosan is often used in association with negatively charged GAGs and other polyanions to form ionic complexes (Suh \& Matthew, 2000). Polysaccharides from GAG family, such as hyaluronic acid (HA) (Ansboro et al., 2014) and chondroitin sulfate (Lim et al., 2011) were also explored as microcarriers for signaling agent delivery because of their natural presence and their crucial functions within the extracellular matrix of connective tissue. However, in order to induce gel formation, chemical modifications of these polysaccharides are required. The toxicity of cross-linking molecules as well as non-degradable cross-linking setting must therefore be considered and may become limiting factors in biological applications. Thus, the research of new molecules that can easily be structured into microgels for bioactive compounds delivery and which are endowed with biological activities (e.g. similar to GAGs) is still encouraged.

HE800 exopolysaccharide (EPS) is an innovative polysaccharide secreted by deep-sea hydrothermal bacteria Vibrio diabolicus, which displays interesting biological activities resulting from its unique structure (Raguénès, Christen, Guezennec, Pignet, \& Barbier, 1997; Rougeaux, Kervarec, Pichon, \& Guezennec, 1999; Zanchetta, Lagarde, \& Guezennec, 2003; Senni et al., 2013). HE800 EPS is a linear non-sulfated polysaccharide of a tetrasaccharidic repeating unit composed of $\mathrm{N}$-acetylglucosamine (GlcNAc), two glucuronic acids (GlcA) and $\mathrm{N}$-acetyl-galactosamine (GalNAc) covalently linked in the following sequence: $\rightarrow 3)-\beta-D-G l c p N A c-(1 \rightarrow 4)-\beta-D-G l c p A-(1 \rightarrow 4)-\beta-D-$ GlcpA-(1 $\rightarrow 4)-\alpha-D-G a l p N A c-(1 \rightarrow$ (Rougeaux, Kervarec, Pichon, \& Guezennec, 1999). This unusual structure presents some similarities to the HA structure, which contains alternating GlcNAc and GlcA residues linked by $\beta-(1 \rightarrow 4)$ and $\beta-(1 \rightarrow 3)$ bonds (Atkins \& Sheehan, 1971). Besides its HA-like structure, native HE800 EPS, either in dry or in soluble state, was demonstrated to possess GAG-like properties since it enhanced in vivo bone regeneration (Zanchetta, Lagarde, \& 
Guezennec, 2003) and stimulated collagen structuring as well as extracellular matrix settle by fibroblasts in reconstructed dermis (Senni et al., 2013). Both structural and functional features of HE800 EPS could therefore be explored to elaborate new microcarriers for biological molecule delivery that can further be used in tissue engineering applications.

When developing microgel-based delivery systems, it is important to properly control the particle size and size distribution, which both highly influence release kinetics. Microgels are usually produced by emulsification methods and different techniques are applied, e.g. dripping, jetting, sonication (Funduneanu, Nastruzzi, Carpov, Desbrieres, \& Rinaudo, 1999). However, none of these techniques allows to obtain microgels with a narrow size distribution and permits to encapsulate the totality of the bioactive species. Moreover, the use of high energy and temperature may lead to alteration of the polysaccharide structure and to degradation of the biological molecules to be encapsulated within the microgels. Taking into account all these parameters, microfluidic technology, manipulating multiphase laminar flows to produce homogeneous structures, appeared as a versatile method for generating micrometer-sized droplets with controllable size and functionality (Zhang, Tumarkin, Sullan, Walker, \& Kumacheva, 2007; Marquis, Renard, \& Cathala, 2012; Marquis, Davy, Fang, \& Renard, 2014). Another incontestable advantage of microfluidics is the possibility to produce microstructures and, at the same time, to encapsulate the totality of the bioactive molecules, in one-step process. This process conducts to a homogeneous distribution of these molecules within microgel (Xu et al., 2009). Although highly advantageous, the use of microfluidic technology to produce microgels for bioactive compound encapsulation has not completely been explored yet as few studies were only reported (Xu et al., 2009; Chen et al., 2013). In the present work, HE800 EPS, innovative polysaccharide from marine origin displaying both GAG-like structure and biological properties has been used for the first time to generate microgels that could further be used as carriers for bioactive molecule delivery, especially in regenerative medicine field, e.g. to reconstruct bone or cartilage tissues. Indeed, when embedded into hydrogel scaffold, these composite carriers are expected to support cell morphogenesis and 
123 proliferation through a sustained delivery of encapsulated compounds. HE800 EPS was physically

124 structured in the presence of divalent cations into microparticles and microfibers using

125 microfluidics. In order to optimize the experimental microfluidic parameters, numerical simulations

126 were developed as predictive tools. The influence of the polysaccharide concentration and its

127 molecular weight on the microparticle morphology was assessed. Furthermore, the use of these

128 microgels as vehicles for sustained delivery of a model protein, namely Bovine Serum Albumin

129 (BSA), was evaluated and the protein release was studied.

130

\section{Materials and Methods}

132

133

134

135

136

137

138

139

140

141

142

143

144

145

146

147

\subsection{Production of the native HE800 EPS and HE800 derivatives, and their characterization.}

HE800 EPS is naturally produced under controlled conditions by fermentation of a nonpathogenic marine bacteria, Vibrio diabolicus, HE800 strain (CNCM: I-1629), isolated in a deep sea hydrothermal vent in the East Pacific Rise from a polychaete annelid Alvinella pompejana. HE800 EPS was produced as previously described (Raguénès, Christen, Guezennec, Pignet, \& Barbier, 1997; Rougeaux, Kervarec, Pichon, \& Guezennec, 1999) using a 2L fermenter containing 1L of marine 2216 broth medium supplemented with glucose, at atmospheric pressure, at $25^{\circ} \mathrm{C}$ and $\mathrm{pH}$ 7.2. HE800 derivatives (HE800 DRs) were obtained by a free-radical depolymerization process (Senni et al., 2008). Briefly, native EPS was dissolved in water at $7 \mathrm{wt} \%$ and $2 \mathrm{~g}$ of $\left(\mathrm{CH}_{3} \mathrm{COO}\right)_{2} \mathrm{Cu}$ were added. The resulting mixture was then heated at $60^{\circ} \mathrm{C}$ and the $\mathrm{pH}$ was set at 7.5. A diluted $\mathrm{H}_{2} \mathrm{O}_{2}$ solution was continuously added $(1 \mathrm{~mL} / \mathrm{min})$ to the EPS solution under controlled $\mathrm{pH}$ conditions using a pHstat (Hache and Lange). The polysaccharide chains were stabilized by an overnight room temperature reduction reaction with $\mathrm{NaBH}_{4}$. Excess of $\mathrm{NaBH}_{4}$ was quenched with $\mathrm{CH}_{3} \mathrm{COOH}(10 \mathrm{M})$. Copper cations were chelated on Chelex ${ }^{\circledR} 20$ resin (sodium form). The resulting solution of HE800 derivatives in the form of sodium salts was ultrafiltrated before being freeze-dried. 
148 Sugar composition of the native HE800 EPS and HE800 DRs was determined by gas

149 chromatography analysis of trimethylsilyl derivatives after acidic methanolysis (Kamerling,

150 Gerwing, Vliegenthart, \& Clamp, 1975). The weight-average molecular weight (Mw) was

151 determined by High-Performance Size Exclusion Chromatography (HPSEC) coupled with a multi-

152 angle light scattering detector (MALS, Dawn Heleos-II ${ }^{\mathrm{TM}}$, Wyatt technology) and a differential

153 refractive index (RI) detector (Hitachi L2490). HPSEC system was composed of an HPLC system

154 Prominence Shimadzu ${ }^{\mathrm{TM}}$, a PL aquagel-OH mixed (Varian) guard column, and a PL aquagel-OH

155 mixed (Varian) separation column.

156

157

\subsection{HE800 DR sample preparation for AFM imaging.}

HE800 DR (300 $000 \mathrm{~g} / \mathrm{mol})$ was solubilized overnight at $0.1 \mathrm{wt} \%$ in MilliQ water. The

solution was then diluted at $5 \mu \mathrm{g} / \mathrm{mL}$ in water. $\mathrm{CuCl}_{2}$ solution at $0.5 \mathrm{wt} \%$ was prepared in MilliQ water and was mixed $(1: 1, \mathrm{v} / \mathrm{v})$ with an aqueous HE800 DR solution at $0.1 \mathrm{wt} \%$. The mixture was

161 diluted at $5 \mu \mathrm{g} / \mathrm{mL}$. $10 \mu \mathrm{L}$ of each diluted solution was deposited onto freshly cleaved mica surface and dried at room temperature prior to AFM observation.

163

2.3. Gelling properties of HE800 DRs in the presence of divalent cations.

Gelling ability of HE800 DRs was assessed by mixing (1:1, v/v) in a glass tube an aqueous solution of $\mathrm{HE} 800 \mathrm{DR}$ at $2 \mathrm{wt} \%\left(\mathrm{pH} \mathrm{7)}\right.$ with an aqueous solution of divalent salts: $\mathrm{CaCl}_{2}, \mathrm{CuCl}_{2}$,

$167 \mathrm{MgCl}_{2}, \mathrm{ZnCl}_{2}$ at $1 \mathrm{wt} \%$. To evaluate gelation, tubes were immediately reversed.

168

169

170

171

172

173

\subsection{Fabrication of the microfluidic Flow-Focusing Devices (FFDs).}

Microfluidic devices were prepared using PDMS (RTV 615, Elecoproduit, France) and a soft lithography technique (Xia \& Whitesides, 1998). SU-8 2100 (CTS, France) positive-relief 
174 molds were produced on silicon wafers (Si-mat, Germany). To produce the microfluidic chip,

175 PDMS mixture (10:1 base polymer/curing agent) cast from the mold was placed on a thin layer of

176 PDMS mixture (20:1 base polymer/curing agent) previously poured and fixed in a Petri dish. The

177 cross-linker diffused as a result of the PDMS gradient. The chip was then oven-treated at $60^{\circ} \mathrm{C}$ for

$17824 \mathrm{~h}$ to strengthen the cross-linking. The microchannels were rectangular in shape with a uniform

179 height of $130 \mu \mathrm{m}$. Channel dimensions were determined by profilometry.

180

\subsection{Generation of HE800 microassemblies using microfluidics.}

Microfluidic three-channel FFD with three inlets delivering the dispersed phase: $\mathrm{CuCl}_{2}$ at 1

wt $\%$ (inlet 1, I1), water (inlet 2, I2) and an aqueous solution of HE800 DR at 1\%, $1.5 \%$ or 2 wt\%

(pH 7) (inlet 3, I3), and one inlet for the continuous phase: sunflower seed oil (Fluka) + Span 80 at

1 wt\% (inlet 4, I4) was designed to produce the microparticles (Fig. S1, Supplementary data).

Teflon tubing connected the syringes with the inlets of the device and the outlet of the device with a tube containing $0.4 \mathrm{~mL}$ of $\mathrm{CuCl}_{2}$ solution at $1 \mathrm{wt} \%$. Digitally controlled syringe pumps (Harvard Apparatus PHD 2000) delivered all liquid phases to the microfluidic device. The following flow rates of the dispersed phase: $\mathrm{HE} 800 \mathrm{DR}$ at $2 \mu \mathrm{L} / \mathrm{min}, \mathrm{CuCl}_{2}$ at $3 \mu \mathrm{L} / \mathrm{min}$ and water at $4 \mu \mathrm{L} / \mathrm{min}$, and of the continuous phase: oil at $35 \mu \mathrm{L} / \mathrm{min}$ were applied during the experiment. The system was run

191 for 20 min per tube. The microparticles suspension was washed three times with $\mathrm{CuCl}_{2}$ solution at 1

192 wt \% and stored in $\mathrm{CuCl}_{2}$ solution at $4^{\circ} \mathrm{C}$ for further studies. Microfluidic two-channel FFD with two

193 inlets delivering the dispersed phase: aqueous solutions of $\mathrm{CuCl}_{2}$ at $1 \mathrm{wt} \%$ (I1) and $\mathrm{HE} 800 \mathrm{DR}$ at 2 $194 \mathrm{wt} \%(\mathrm{pH} 7)(\mathrm{I} 2)$, and one inlet for the continuous phase: sunflower seed oil + Span 80 at 1 wt\% (I3) was elaborated to produce the microfibers (Fig. S2, Supplementary data). The flow rates of the disperse phase were as follows: $\mathrm{HE} 800 \mathrm{DR}$ at $2 \mu \mathrm{L} / \mathrm{min}$ and $\mathrm{CuCl}_{2}$ at $3 \mu \mathrm{L} / \mathrm{min}$, while the flow rate of the continuous oil phase was maintained at $3 \mu \mathrm{L} / \mathrm{min}$.

\subsection{Numerical simulations.}


Numerical simulations of the droplet formation process were conducted with Comsol

201

202

203

204

205

206

207

208

209

210

211

212

213

214

215

216

217

218

219

220

221

222

223

224

225

Multiphysics 4.3a. The level-set method implemented in the laminar two-phase flow model was used to track the droplet interface during its formation. Both the geometry and the dimensions of the designed microfluidic device, in its top view, were reproduced to form a two-dimensional FFD. This 2D configuration offers the great advantage to considerably reduce the calculation time. The continuous phase (sunflower oil) is pumped through the two side inlets whereas the dispersed phase (aqueous solution of HE800 DR) is injected from the three left channels. These two phases were modeled as incompressible Newtonian fluids and their properties were experimentally obtained: density of the continuous phase $920 \mathrm{~kg} / \mathrm{m}^{3}$, density of the dispersed phase $1000 \mathrm{~kg} / \mathrm{m}^{3}$. The viscosity of two phases was measured using a RheoStress 300 (ThermoHaakes, Germany) rheometer: viscosity of the continuous phase 9e-2 Pa.s, viscosity of the dispersed phase 2e-3 Pa.s. The boundary condition of the PDMS channel walls was defined as "wetted wall" with a contact angle of $108^{\circ}$. The surface tension for the interface between the continuous and dispersed phases was measured at $15 \mathrm{mN} / \mathrm{m}$ using the tensiometer CAM 200 (Helsinki, Finland).

\subsection{BSA-FITC encapsulation in the microparticles using microfluidics.}

To visualize BSA encapsulated in the microparticles, fluorescent dye FITC (Sigma-Aldrich) was grafted to the protein, as previously described (Marquis, Davy, Cathala, \& Renard, 2015). To encapsulate BSA-FITC in the microparticles, the protein was directly solubilized at $0.1 \mathrm{wt} \%$ in the aqueous solution of $\mathrm{HE} 800 \mathrm{DR}$ either at 1 or $2 \mathrm{wt} \% . \mathrm{CuCl}_{2}$ concentration was kept constant at 1 wt $\%$. The BSA-FITC loaded microparticles were produced using three channel FF device with the flow rates of the continuous oil phase of $35 \mu \mathrm{L} / \mathrm{min}$ and the dispersed phases: HE800 DR/BSAFITC of $2 \mu \mathrm{L} / \mathrm{min}, \mathrm{CuCl}_{2}$ of $3 \mu \mathrm{L} / \mathrm{min}$ and water of $4 \mu \mathrm{L} / \mathrm{min}$. BSA-FITC loaded microparticles were collected during $20 \mathrm{~min}$ in tubes containing $0.4 \mathrm{~mL}$ of $\mathrm{CuCl}_{2}$ solution at $1 \mathrm{wt} \%$. Several tubes were collected in the same experimental conditions. Microparticles were centrifuged ( 2 min, 2000 g) and washed three times in water. The final volume of the microparticles was $200 \mu \mathrm{L}$ in water. 
2.8. In vitro BSA-FITC release from the microparticles.

$1 \mathrm{~mL}$ of PBS $20 \mathrm{mM}$ or HEPES $20 \mathrm{mM}$ at pH 7.4 was added to $200 \mu \mathrm{L}$ of the microparticle suspension in water. Samples were incubated at $37^{\circ} \mathrm{C}$ under gentle shaking during 8 days. For different incubation times, $1 \mathrm{~mL}$ of the supernatant was removed and replaced by fresh buffer. The release assays were made in triplicate. The amount of the protein released and recovered in the collected supernatants was measured using fluorescence spectroscopy (Hitachi F-4500). Samples were excited at $480 \mathrm{~nm}$ and fluorescence was recorded between 495 and $650 \mathrm{~nm}$ with $5 \mathrm{~nm}$ slit width and $1 \mathrm{~s}$ response time. The cumulative protein release was calculated by taking into account the amount of preloaded and released protein. The error bars correspond to the experimental dispersion.

\subsection{Microscopy imaging.}

Phase contrast and fluorescence microscopy images were captured with an Olympus IX51 inverse microscope equipped with a digital camera (Sony, SCD-SX90). The size distributions of microstructures produced were analyzed using the ImageJ freeware v135c. SEM observations on 242 supercritically-dried samples prepared as previously described (Marquis, Davy, Cathala, \& Renard, 243 2015) were made using a Jeol 6400F microscope operating at $3 \mathrm{kV}$ after gold/palladium sample 244 coating. All AFM images were recorded with a NanoWizard® Atomic Force Microscope (JPK, 245 Germany) operating in intermittent contact mode under ambient conditions. A standard rectangular 246 cantilever (Nanosensors NCL-W) was used for imaging, with a free resonance frequency of 165 $247 \mathrm{kHz}$ and a typical spring constant of about $40 \mathrm{~N} / \mathrm{m}$. The radius curvature of the tip was $\sim 10 \mathrm{~nm}$. In 248 order to ensure the reproducibility of the observed morphology, all samples were scanned at least on 249 three different zones. Each sample was investigated using fresh tips previously cleaned by UV250 ozone treatment. The height and width measurements (typically 80-100 measurements per sample) 251 were done using JPK Data Processing software (JPK, Germany). 


\section{Results and discussion}

254

\subsection{Preparation of HE800 derivatives and their gelling properties.}

In order to promote HE800 EPS structuring into microcarriers for protein delivery, native polysaccharide of high-molecular weight $\left(\mathrm{M}_{\mathrm{w}}=1000000 \mathrm{~g} / \mathrm{mol}\right)$ was firstly depolymerized. Two HE800 derivatives (HE800 DRs) of $100000 \mathrm{~g} / \mathrm{mol}$ and $300000 \mathrm{~g} / \mathrm{mol}$ were prepared using freeradical depolymerization (Senni et al., 2008). Constant molar ratio GlcNAc/GlcA/GalNAc of 1/2/1, determined after osidic unit analyses of the native HE800 EPS and the two HE800 DRs, demonstrates that the depolymerization process had no major impact on the polysaccharide structure. In the following step, the two HE800 DRs were tested for their gelling ability in the presence of different divalent cations. $\mathrm{Ca}^{2+}, \mathrm{Cu}^{2+}, \mathrm{Mg}^{2+}$ and $\mathrm{Zn}^{2+}$ were selected for their interesting biological properties (e.g. stimulation of cell proliferation and differentiation, angiogenesis enhancement as well as anti-bacterial and anti-inflammatory effects) (Hoppe, Güldal, \& Boccaccini, 2011). Gelling properties were studied by mixing (1:1, v/v) an aqueous solution of HE800 DR at 2 wt $\%$ with an aqueous solution of divalent cations at $1 \mathrm{wt} \%$ at $\mathrm{pH}$ 7. It was observed that only $\mathrm{Cu}^{2+}$ ions were able to instantaneously induce HE800 DR gelation. The presence of two consecutive GlcA residues in HE800 sequence most likely favors the formation of stable junction zones between dissociated carboxyl groups of two polysaccharide chains and copper ions (Fig. 1), in a similar way as described for "egg-box" model (Grant, Morris, Rees, Smith, \& Thom, 1973). In the case of alginate and pectin, the two-stage gelation mechanism in the presence of calcium ions was proposed, where the formation of strongly linked dimer associations is followed by the formation of weak inter-dimer associations (Braccini \& Pérez, 2001). The formed junction zones are stabilized not only by cation mediated electrostatic interactions, but other contributions such as van der Waals interactions and hydrogen bonding are also involved. The higher affinity of HE800 DR toward $\mathrm{Cu}^{2+}$ ions in comparison to other ions tested is in agreement with the previous studies on alginate and pectin. In the case of alginate, its affinity to divalent cations has been shown to decrease in the 
278 following order: $\mathrm{Pb}^{2+}>\mathrm{Cu}^{2+}>\mathrm{Cd}^{2+}>\mathrm{Ba}^{2+}>\mathrm{Sr}^{2+}>\mathrm{Ca}^{2+}>\mathrm{Co}^{2+}>\mathrm{Zn}^{2+}>\mathrm{Mn}^{2+}$ (Smidsrød, \& 279 Skjåk-Bræk, 1990). Similar results were also reported for pectin affinity toward various divalent 280 cations (Ouwerx, Velings, Mestdagh, \& Axelos, 1998).

281 Fig. 1. Hypothetical junction zone formed between two HE800 EPS chains cross-linked with a 282 copper ion.

The effect of $\mathrm{Cu}^{2+}$ addition on HE800 DR (300 $\left.000 \mathrm{~g} / \mathrm{mol}\right)$ structuring was assessed using Atomic Force Microscopy (AFM). In order to follow the initial step of gelation mechanism, i.e. first interchain associations before a dense network formation, AFM imaging was done on highly diluted systems. Fig. 2 presents typical height images of HE800 DR (300 $000 \mathrm{~g} / \mathrm{mol})$ fibers in their native

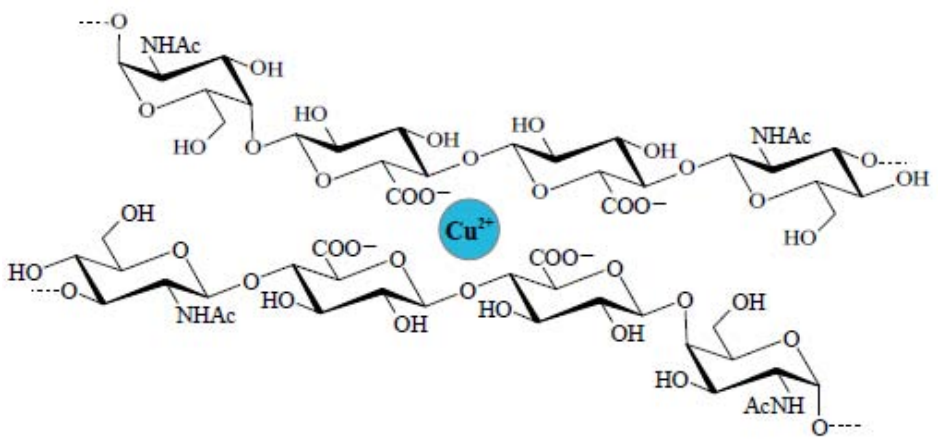

state (Fig. 2A) and after incubation in the presence of $\mathrm{Cu}^{2+}$ ions at $0.5 \mathrm{wt} \%$ (Fig. 2B). For the native polysaccharide, elongated fibers were observed. Height measurements done on the native fibers allowed to determine the average height value of $0.65 \pm 0.15 \mathrm{~nm}$ (Fig. 2C), corresponding to the diameter of a single monomolecular chain. The obtained value is in agreement with the height values determined by AFM for other polysaccharides, such as hyaluronic acid $(0.6 \mathrm{~nm})$ or pectin $(0.5 \mathrm{~nm})$ (Abu-Lail \& Camesano, 2003). In contrast to height measurements, width measurements are affected by tip broadening effects and the values obtained are thus overestimated (Gunning, Kirby, Morris, Wells, \& Brooker, 1995). However, in regarding the high ratio between the average width value of $23.5 \pm 4.4 \mathrm{~nm}$ (Fig. 2D) and the measured diameter, it could be assumed that several chains are laterally associated and constitute the observed native fiber. These lateral chain-chain 
associations appearing already in the native fiber are most likely mediated by residual ions remaining in the sample and by water-mediated hydrogen bonds, which prevent the complete chain dissociation during sample solubilization. The addition of $\mathrm{Cu}^{2+}$ cations to the native HE800 DR (300 $000 \mathrm{~g} / \mathrm{mol}$ ) fibers has considerably affected their morphology. An increase in the average height (3.8 $\pm 0.25 \mathrm{~nm}$, Fig. 2C) as well as the average fiber width $(52.5 \pm 5.0 \mathrm{~nm}$, Fig. 2D) was observed. The increase in both height and width measurements indicates that more chains were associated after $\mathrm{Cu}^{2+}$ addition. This increase demonstrates that copper ions induce chain-chain associations, which result in fine in gel formation when the polysaccharide and ion concentrations are high enough. Similar AFM results were also reported in the case of pectin gelling mechanism investigated in the presence of calcium ions (Zykwinska, Gaillard, Boiffard, Thibault, \& Bonnin, 2009).
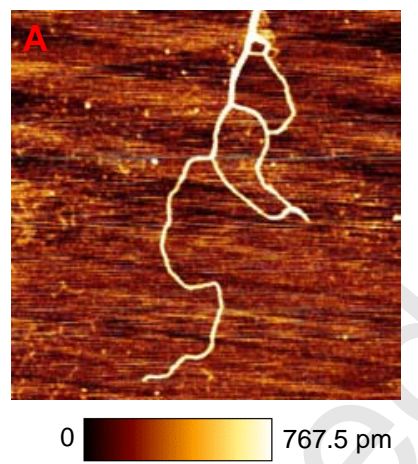
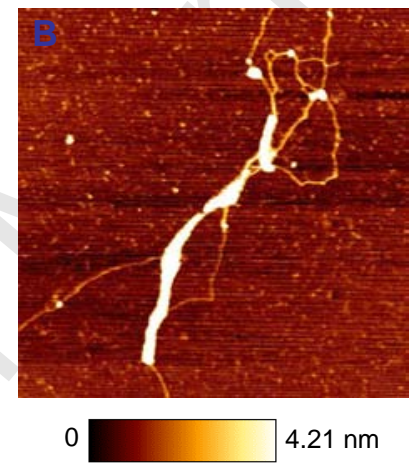
$4.21 \mathrm{~nm}$

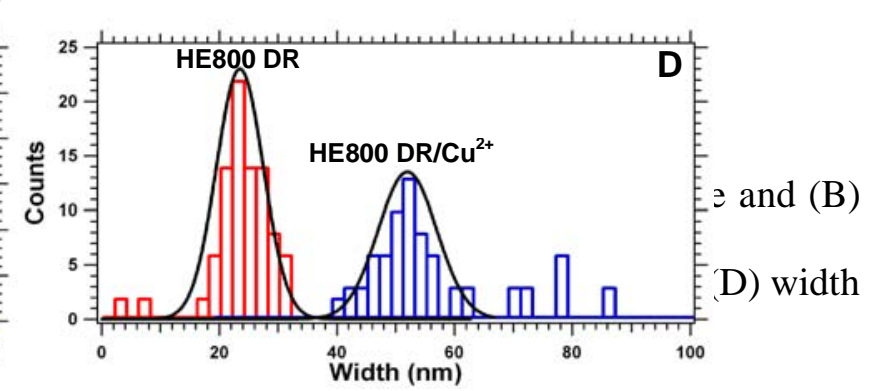

3.2. HE800 DR microassembly formation using microfluidics: numerical simulations and experiments.

Two microfluidic Flow-Focusing Devices (FFDs) were designed to generate HE800 DR microassemblies. A three-channel FFD presented on the Fig. 3A was elaborated to produce the microparticles. The dispersed phase was composed of aqueous solutions of HE800 DR (300 000 
$\mathrm{g} / \mathrm{mol})$ at $2 \mathrm{wt} \%(\mathrm{pH} \mathrm{7})$ and $\mathrm{CuCl}_{2}$ at $1 \mathrm{wt} \%$, whereas the continuous phase was constituted of

322

323

324 sunflower oil. In order to avoid gelling before FFD junction, the water flow was introduced between the polysaccharide and $\mathrm{CuCl}_{2}$ flows. In the designed FFD, the continuous phase is pumped through the two side channels and the dispersed phase is injected from the three left channels (Fig. 3A). Before the experiments, the emulsion within the microfluidic device was modeled by combining the incompressible Navier-Stokes equation, a continuity equation and a level set equation. Precisely, a level set method was implemented to track the interface evolution between the continuous and dispersed phases during the droplet formation (Olsson \& Kreiss, 2005). In this method, the interface is defined by an isocontour curve of the level set function, , equal to 0.5 . The continuous phase is represented by $>0.5$ whereas $<0.5$ corresponded to the dispersed phase.

Numerical simulations were used as predictive tools to optimize the experimental microfluidic parameters (i.e. flow rate and viscosity of the continuous and dispersed phases) for forming monodisperse droplets in the FFD. The droplet size is mainly governed by the competition between the Laplace pressure (proportional to the interfacial tension) and the shear forces exerted on the interface by the continuous phase (Thorsen, Roberts, Arnold, \& Quake, 2001). This force balance is usually expressed by the capillary number $(\mathrm{Ca})$ defined by the product of the viscosity and the velocity of the continuous phase divided by the interfacial tension (Cristini \& Tan, 2004). During an experiment, $\mathrm{Ca}$ directly depends on the velocity of the continuous phase (i.e. flow rate), since the dimensions of the microfluidic device as well as the viscosity and the interfacial tension remain unchanged. Although the velocity of the dispersed phase is not directly included in the $\mathrm{Ca}$ expression, it strongly influences the droplet shape by affecting its breaking process. Therefore, numerical results are shown in terms of capillary number and flow ratio, $Q$, of the continuous phase to the dispersed one (Cristini \& Tan, 2004; Garstecki, Fuerstman, Stone, \& Whitesides, 2006). In Fig. 3B-3D, numerical simulations reveal the successive steps of droplets formation by following the evolution of isocontour curves $(=0.5)$. For the used $C a$ and $Q$ values $(C a=0.01 ; Q=15)$, when the dispersed phase reaches the orifice entrance, a cone-shape with a neck is formed due to 
the flow rate of the continuous phase (Fig. 3B). Then, the shear forces exerted on the interface by

348 the continuous phase become sufficiently strong to pinch off the dispersed phase and conduct to the

349 formation of tiny beads (Fig. 3C). Finally, water-in-oil monodisperse droplets of $100 \mu$ m-diameter 350 are formed (Fig. 3D). Simulations also reveal that the diameter of monodisperse droplets can be 351 easily tuned by playing with $C a$ and $Q$ values. For instance, the increase of $C a$ can induce a 352 significant decrease in the droplet size (Fig. S3, Supplementary data).
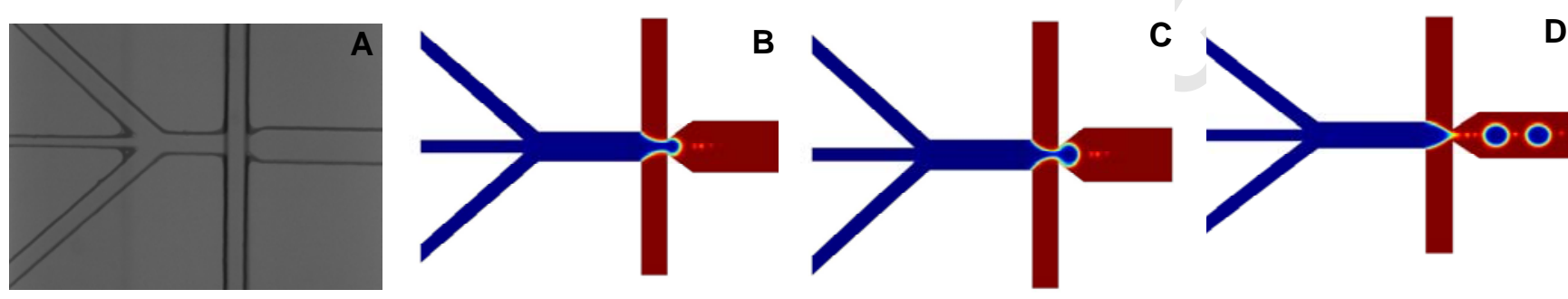

Fig. 3. (A) Experimental three-channel FFD used to generate microparticles. (B-D) Snapshots of droplet formation mechanisms with $C a=0.01$ and $Q=15$. The interface corresponds to an isocontour curve of $=0.5$, while the continuous and the dispersed phases are represented in red $(>0.5)$ and blue $(<0.5)$, respectively.

For experiments, to control the flows of aqueous phases and to follow the gelation inside the droplets formed after FFD junction, fluorescein isothiocyanate (FITC, $0.01 \mathrm{wt} \%$ ) was solubilized in HE800 DR solution (Fig. 4A). As seen on the Fig. 4B, gelling has started in the droplets and was mainly governed by the convection phenomenon (Sarrazin, Bonometti, Prat, Gourdon, \& Magnaudet, 2008). Monodisperse gelled microparticles (Fig. 4C) were produced using the following flow rates of the dispersed (HE800 DR at $2 \mu \mathrm{L} / \mathrm{min}, \mathrm{CuCl}_{2}$ at $3 \mu \mathrm{L} / \mathrm{min}$ and water at 4 $\mu \mathrm{L} / \mathrm{min}$ ) and continuous (oil at $35 \mu \mathrm{L} / \mathrm{min}$ ) phases. The average microparticle diameter was of 102 $\mu \mathrm{m}($ c.v. $=5 \%)$, which is in agreement with the diameter of droplets obtained using numerical
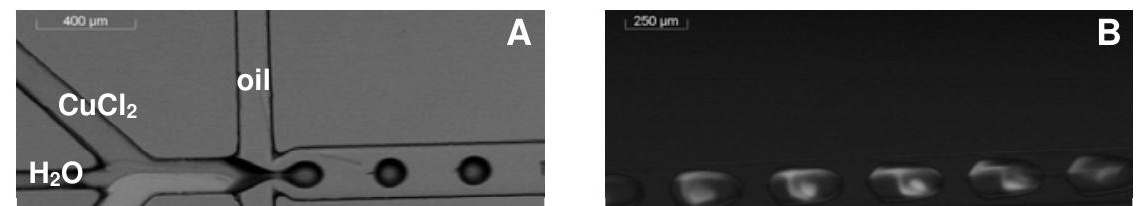
structured in the rose-like form (Fig. 4D), resulting most likely from roll up of the microfiber formed in the droplet, initiated by convective intermixing.
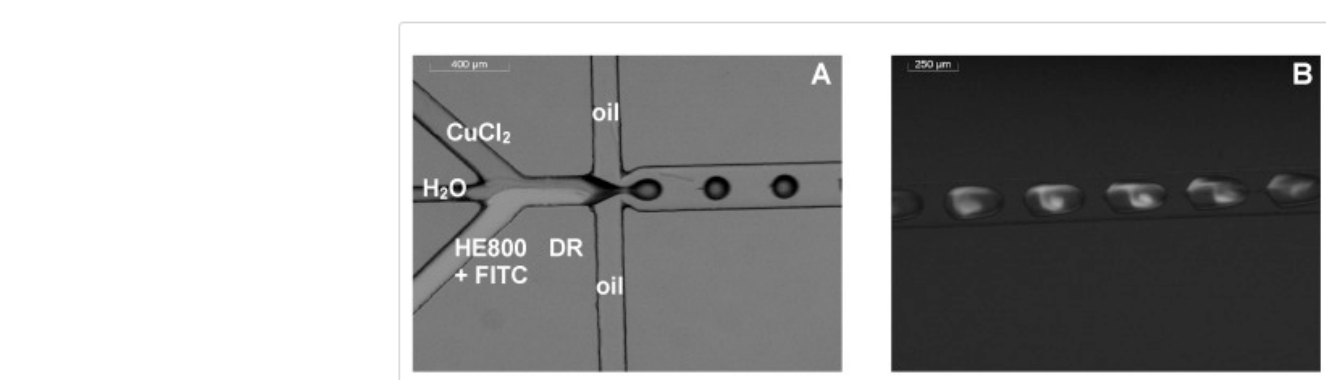

C
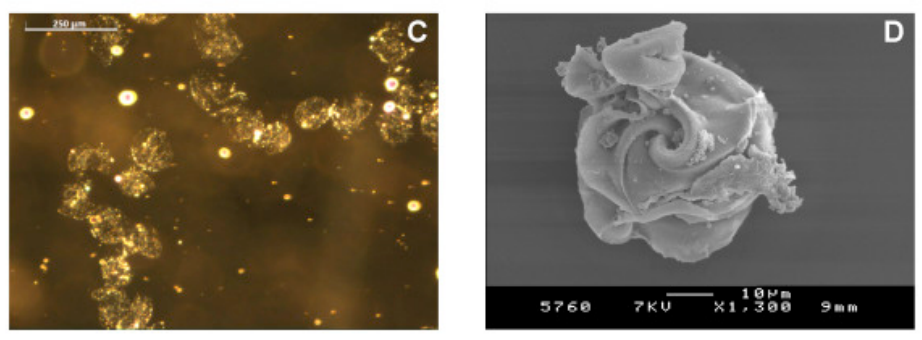

Fig. 4. (A) Three-channel FFD used for microparticle generation. Microparticles formed with HE800 DR (300 $000 \mathrm{~g} / \mathrm{mol})$ at $2 \mathrm{wt} \%$ and $\mathrm{CuCl}_{2}$ at $1 \mathrm{wt} \%$ observed by (B) fluorescence microscopy, showing a convection phenomenon inside the microdroplets, (C) optical microscopy, showing freshly collected gelled microparticles and (D) SEM, showing a typical rose-like structure obtained.

It was shown furthermore that HE800 DR (300 $000 \mathrm{~g} / \mathrm{mol})$ can also be structured in microfibers in the presence of $\mathrm{Cu}^{2+}$ ions. For microfiber generation, two-channel FFD presented on the Fig. 5A was designed. In this case, the dispersed phase was composed of aqueous solutions of HE800 DR (300 $000 \mathrm{~g} / \mathrm{mol}$ ) at $2 \mathrm{wt} \%$ with FITC at $0.01 \mathrm{wt} \%$ and $\mathrm{CuCl}_{2}$ at $1 \mathrm{wt} \%$. Contrary to the microparticles, the stable cylindrical stream leading to microfibers was obtained by decreasing the flow rate of the continuous oil phase at $3 \mu \mathrm{L} / \mathrm{min}$, while the flow rate of the disperse phases was kept constant (HE800 DR at $2 \mu \mathrm{L} / \mathrm{min}$ and $\mathrm{CuCl}_{2}$ at $3 \mu \mathrm{L} / \mathrm{min}$ ). The microfibers of 1 to $5 \mathrm{~mm}$ in length and of 100 $\mu \mathrm{m}(\mathrm{c.v.}=7 \%)$ in diameter were obtained (Fig. 5B). SEM images revealed the smooth surface of the microfibers formed (Fig. 5C). 

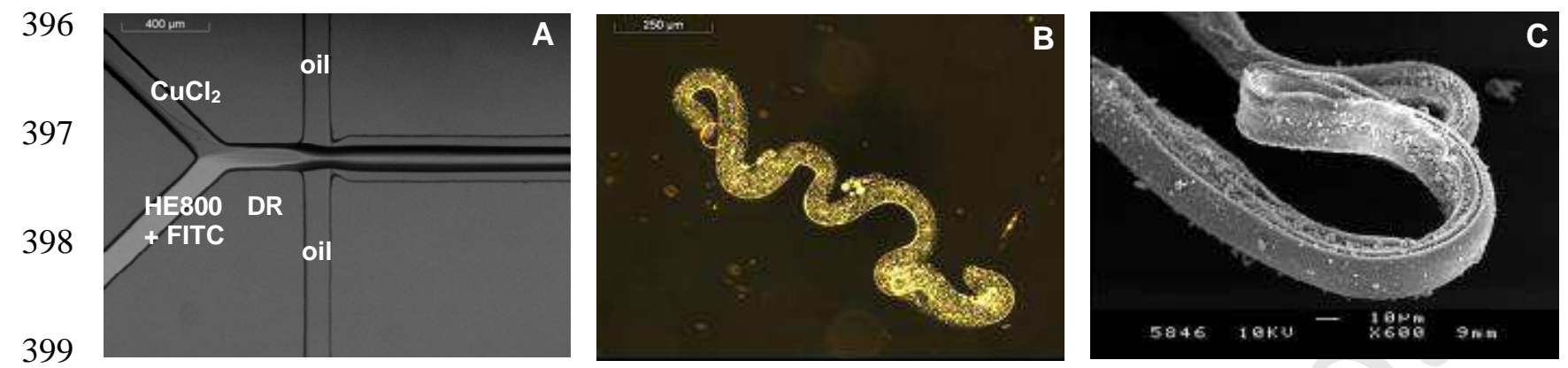

Fig. 5. (A) Two-channel FFD used for microfiber generation. Microfibers formed with HE800 DR (300 $000 \mathrm{~g} / \mathrm{mol}$ ) at $2 \mathrm{wt} \%$ and $\mathrm{CuCl}_{2}$ at $1 \mathrm{wt} \%$ observed by (B) optical microscopy, showing the typical freshly collected gelled microfiber and (C) SEM, showing a typical morphology of the microfibers formed.

3.3. The influence of HE800 DR concentration and molecular weight on the microparticle morphology.

In order to evaluate if HE800 DR $(300000 \mathrm{~g} / \mathrm{mol})$ concentration had an impact on the 407 microparticle morphology, microparticles were generated using two lower polysaccharide 408 concentrations of either $1.5 \%$ or $1 \mathrm{wt} \%(\mathrm{pH} 7)$. The $\mathrm{CuCl}_{2}$ concentration was kept constant at 1 $\mathrm{wt} \%$. The flow rates of the continuous and dispersed phases were the same as for the microparticles produced with HE800 DR at 2 wt\%. SEM images presented on the Fig. 6 (A and B) revealed that decreasing polysaccharide concentration led to a progressive loss of the rose-like morphology observed at $2 \mathrm{wt} \%$ (Fig. 4D). Moreover, microparticles formed at $1 \mathrm{wt} \%$ were completely devoid of

413 this peculiar structure and appeared more homogeneous. This morphology results most likely from 414 more homogeneous mixing between polysaccharide chains and copper ions induced by convection 415 phenomenon before gelation. In contrast, for higher polysaccharide concentration $(1.5 \%$ and 2 $416 \mathrm{wt} \%)$, gel formation occurs instantaneously when the flow of the polysaccharide meets the copper 417 flow, which leads to the rose-like structure. It was assessed furthermore that the microparticle 418 morphology can also be affected by the polysaccharide molecular weight. At the same 419 polysaccharide concentration ( $2 \mathrm{wt} \%$ ), the morphologies of the microparticles formed were 
420

421

422

423

424

425

426

427

428

429

430

431

432

433

434

440

441

442

443

444

445

different. HE800 DR of $300000 \mathrm{~g} / \mathrm{mol}$ was structured in the rose-like form (Fig. 4D), whereas the morphology of the microparticles formed with HE800 DR of lower molecular weight (100 000 g/mol) appeared more homogeneous (Fig. 6C). This morphology was similar to the one obtained with the derivative of $300000 \mathrm{~g} / \mathrm{mol}$ at $1 \mathrm{wt} \%$ (Fig. 6B). It is likely that the gelation rate decreases with shorter chains, which allows more efficient mixing of the polysaccharide chains and copper ions before gelling.

$300000 \mathrm{~g} / \mathrm{mol}, 1.5 \mathrm{wt} \%$

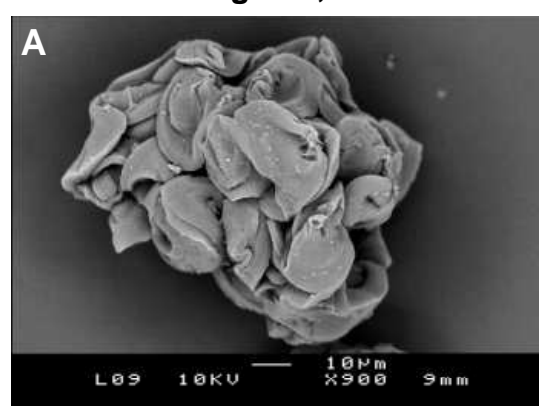

$300000 \mathrm{~g} / \mathrm{mol}, 1$ wt\%

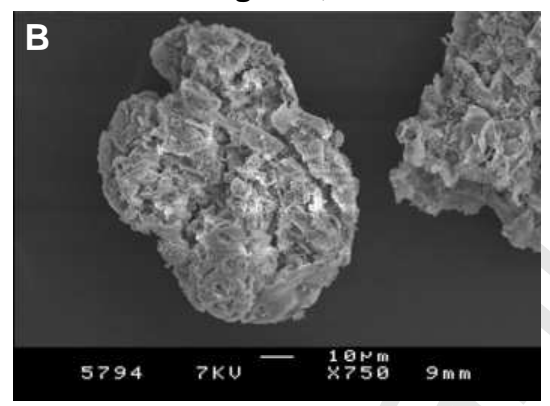

$100000 \mathrm{~g} / \mathrm{mol}, 2 \mathrm{wt} \%$

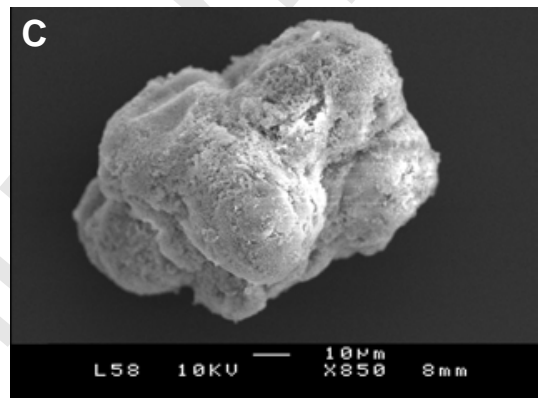

Fig. 6. Microparticles formed with HE800 DR (A) $300000 \mathrm{~g} / \mathrm{mol}$ at $1.5 \mathrm{wt} \%$, (B) $300000 \mathrm{~g} / \mathrm{mol}$ at $1 \mathrm{wt} \%$ and (C) $100000 \mathrm{~g} / \mathrm{mol}$ at $2 \mathrm{wt} \%$, observed by SEM.

3.4. Model protein encapsulation and its release from the microparticles.

The potential application of the microgel particles formed with HE800 DR (300 $000 \mathrm{~g} / \mathrm{mol})$ physically cross-linked with $\mathrm{Cu}^{2+}$ ions as a protein delivery system was assessed by encapsulating a model protein, namely Bovine Serum Albumin labeled with fluorescein isothiocyanate (BSAFITC). Indeed, BSA-FITC has already been used as a model protein in several in vitro and in vivo delivery studies (Koppolu et al., 2014; Marquis, Renard, \& Cathala, 2012; Kim et al., 2009; Pessi et al., 2014). BSA is a large protein of a molecular weight of $69 \mathrm{kDa}$ and it is composed of $14 \%$ of basic and $18 \%$ of acidic groups, with a pI of 4.8 (Coradin, Coupé, \& Livage, 2003). The microparticle formation and the protein encapsulation were done in one-step using microfluidic three-channel FFD shown on the Fig. 4A. Indeed, the advantage of microfluidics is that the whole protein is integrated within microparticles, which are collected in their gelled and thus stabilized form at the end of the microfluidic chip. Moreover, the use of mild conditions to induce HE800 gelation, i.e. physical cross-linking, should preserve the activity of the encapsulated protein, as 
reported for alginate and pectin (Jay, \& Saltzman, 2009; Marquis, Davy, Cathala, \& Renard, 2015).

In order to produce protein loaded microparticles, the dispersed phase was composed of water and aqueous solutions of HE800 DR (300 $000 \mathrm{~g} / \mathrm{mol})$ containing BSA-FITC at $0.1 \mathrm{wt} \%(\mathrm{pH} 7)$ and $\mathrm{CuCl}_{2}$ at $1 \mathrm{wt} \%$. To assess if the polysaccharide concentration had an impact on protein release from the microparticles, two polysaccharide concentrations of $1 \%$ and $2 \mathrm{wt} \%$ were tested to elaborate the microparticles. As previously shown on SEM images, more homogeneous structure was obtained at a concentration of $1 \mathrm{wt} \%$ (Fig. 6B), instead of heterogeneous one (rose-like structure) formed at 2 wt\% (Fig. 4D). These two distinct morphologies suggest that the gelling ratio differs within both structures, which may in turn influence the protein distribution and its further release. To ensure that the same amount of the protein was encapsulated within microparticles formed at HE800 DR concentrations of $1 \%$ and $2 \mathrm{wt} \%$, the flow rates of the continuous phase (oil at $35 \mu \mathrm{L} / \mathrm{min}$ ) and the dispersed phases (HE800 DR at $2 \mu \mathrm{L} / \mathrm{min}, \mathrm{CuCl}_{2}$ at $3 \mu \mathrm{L} / \mathrm{min}$ and water at $4 \mu \mathrm{L} / \mathrm{min}$ ) were kept constant. The fluorescence microscopy images presented on the Fig. 7A show that the protein was successfully encapsulated within microparticles formed at polysaccharide concentrations of $1 \%$ and $2 \mathrm{wt} \%$. SEM images of the protein loaded microparticles are presented on the Fig. 7B. It was noticed that the morphologies of BSA loaded microparticles differ from those obtained before incorporating the protein into the system. In particular, the microparticle formed with HE800 DR at 2 wt\% started to loose its rose-like structure. This observation put forward that the protein encapsulation has disrupted the polysaccharide gelling and affected its gelling ratio due most likely to steric hindrance and/or polysaccharide-protein interactions. Indeed, even if at pH 7, HE800 DR and BSA-FITC are both negatively charged, the presence of $\mathrm{Cu}^{2+}$ ions would decrease the repulsive forces between both entities, especially if BSA bears only $18 \%$ of acidic groups. In addition, attractive hydrophilic and hydrophobic forces may also be responsible for protein-polysaccharide interactions.

(A)
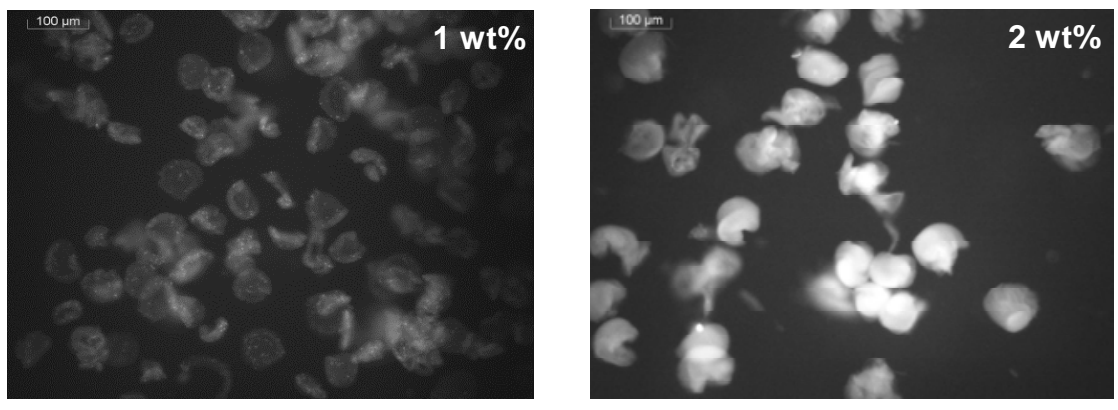

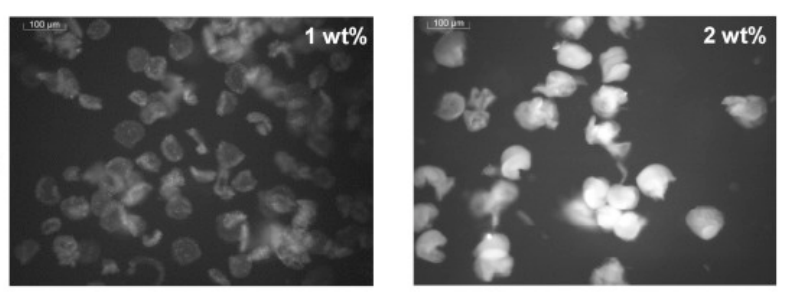

(B)
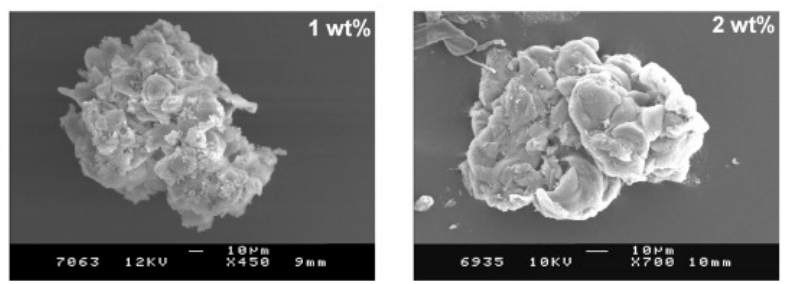

476

(C)

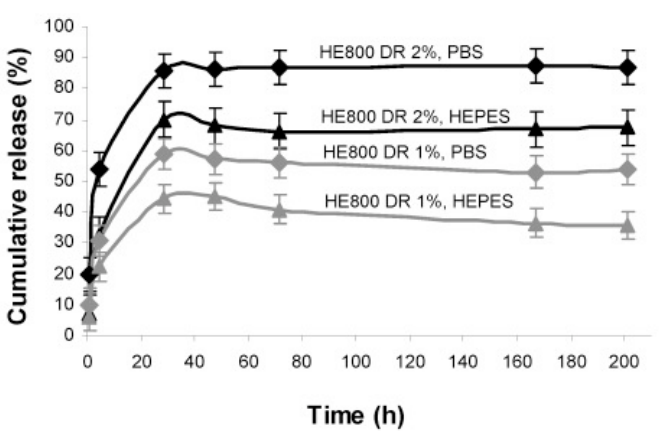

Fig. 7. BSA-FITC loaded microparticles formed with HE800 DR (300 $000 \mathrm{~g} / \mathrm{mol})$ at $1 \%$ and $2 \mathrm{wt} \%$ observed by (A) fluorescence microscopy and (B) SEM. Cumulative release profiles of BSA-FITC released from the microparticles formed with HE800 DR (300 $000 \mathrm{~g} / \mathrm{mol})$ at $1 \%$ and $2 \mathrm{wt} \%$ and incubated in either PBS or HEPES buffers at $37^{\circ} \mathrm{C}$ at $\mathrm{pH} 7.4(\mathrm{C})$.

The release of the protein loaded within HE800 DR/ $\mathrm{Cu}^{2+}$ microparticles was studied at $37^{\circ} \mathrm{C}$. The microparticles were incubated in two buffers that are frequently used for cell culture, namely PBS and HEPES at pH 7.4. The release profiles of BSA-FITC from microparticles formed with HE800 DR at $1 \%$ and $2 \mathrm{wt} \%$ are shown on the Fig. $7 \mathrm{C}$. The cumulative percentages of protein release are presented up to $200 \mathrm{~h}$. The release kinetics were highly affected by either the polysaccharide concentration used to elaborate the microparticles or the buffer tested. Release profiles obtained for

491 the protein encapsulated within microparticles formed with HE800 DR at $1 \%$ were characterized by 492 an initial burst of 6-22\% in HEPES and 10-30\% in PBS, followed by a slow sustained release 
493

494

495

496

497

498

499

500

501

502

503

504

505

506

507

508

509

510

511

512

513

514

515

516

517

518

leading to a maximum protein release of $44 \%$ in HEPES and $59 \%$ in PBS. In contrast, higher amounts of protein were liberated from microparticles structured with HE800 DR at 2 wt $\%$. An initial burst of 7-33\% in HEPES and 20-54\% in PBS was measured, followed by a sustained release with a plateau value reached within $28 \mathrm{~h}$ corresponding to a maximum protein release of $70 \%$ in HEPES and $85 \%$ in PBS. The important difference in protein release between BSA-FITC loaded microparticles structured with HE800 DR at $1 \%$ and $2 \mathrm{wt} \%$ is most likely due to the fact that at 2 $\mathrm{wt} \%$, the gelling ratio remains higher than that at lower polysaccharide concentration of $1 \mathrm{wt} \%$.

More rapid gelling leads to less homogeneous morphology, as observed for the microparticles prepared with HE800 DR at $2 \mathrm{wt} \%$ in comparison to those formed with HE800 DR at $1 \mathrm{wt} \%$.

Gelling ratio influences the protein distribution inside the microparticle and more homogeneous distribution should be obtained in the microparticles with lower gelling ratio (HE800 DR at $1 \mathrm{wt} \%$ ).

Better incorporation of the protein inside the microgel structure results in lower initial burst and lower amount of the total protein released. The fact that the protein was not completely released from the microparticles can be explained not only by the homogeneous protein distribution inside the microparticles but also by polysaccharide-protein interactions mediated most likely by hydrophilic-hydrophobic forces. Indeed, the microparticles incubated either in PBS or in HEPES were rapidly swelled after the addition of buffers (Fig. S4, Supplementary data). Consequently, if the protein was not interacting with the polysaccharide matrix, it would be fully released and recovered in the buffer. In fact, HE800 EPS in its hydrated form may act as a protein reservoir by sequestrating the molecules before releasing them, in the same manner as GAGs present within extracellular matrix (Zcharia et al., 2005). Therefore, the presence of soluble HE800 molecules liberated from the swelled microparticles could be highly beneficial in engineered tissues. Indeed, HE800 EPS bioactivity was previously demonstrated on collagen structuring and dermal fibroblast settle in extracellular matrix (Senni et al., 2013).

It was also observed that the protein release depended not only on the polysaccharide concentration but also on the buffer used. Indeed, the amount of the protein released in PBS was $15-20 \%$ higher 
compared to that liberated in HEPES. It is likely that PBS complexes $\mathrm{Cu}^{2+}$ ions, which are

520 physically cross-linked with HE800 DR, in a higher degree than HEPES. Finally, microparticles

521 structured with this unusual polysaccharide displaying GAG-like properties could not only be used

522

523

524

525

526

527

528

529

530

531

532

533

534

535

536

537

538

539

540

541

542

543 as a protein delivery system, but also as local $\mathrm{Cu}^{2+}$ ion delivery system. Indeed, several studies have demonstrated the biological potential of copper ions towards angiogenesis (Gérard, Bordeleau, Barralet, \& Doillon, 2010) and stimulation of mesenchymal stem cell differentiation (Rodríguez, Ríos, \& González, 2002).

\section{Conclusion}

In the present study, an unusual polysaccharide from marine origin, namely HE800 EPS was structured for the first time using microfluidics in functional microcarriers that can be used as protein delivery systems. The significant advantage of the present delivery system is based on peculiar polysaccharide GAG-like structure and its biological properties, which can both be explored to create an innovative biomaterial for tissue engineering applications. This high-added value polysaccharide was shown to be able to form microparticles and microfibers, through physical cross-linking with copper ions, using microfluidics. It was shown that the microparticle morphology could be modulated by the polysaccharide concentration and its chain length, and that either homogeneous or heterogeneous structures could be obtained. The microparticle morphology seems to be a key factor since it highly influenced the protein release. Indeed, a lower amount of BSA protein was released when the protein was encapsulated within homogeneous microparticles in comparison to heterogeneous ones. The potential application of HE800 microgels in bone or cartilage tissue engineering as growth factor delivery system may now be explored. The incorporation of protein loaded HE800 microgels in their wet state into hydrogel scaffolds and the impact of a sustained growth factor release onto mesenchymal stem cell differentiation in vitro and in vivo will be assessed. Depending on the growth factor nature, the binding polysaccharide-protein 
544 affinity will be tuned not only by the polysaccharide concentration but also by the presence of 545 additional charges on the polysaccharide backbone, e.g. sulfate groups.

546 


\section{Acknowledgment} Financial supports were provided by EU Interreg IVA 2 Seas "BioCare Marine". A.Z. and

548 M.M. contributed equally to this work. The authors would like to acknowledge Jacqueline Ratiskol

549 for sugar analysis, Joëlle Davy and Nicolas Stephan for their assistance with SEM microscopy 550 experiments. Marie-Hélène Ropers is acknowledged for interfacial tension measurements. 
551

552

553

554

555

556

557

558

559

560

561

562

563

564

565

566

567

568

569

570

571

572

573

574

575

\section{References}

Abu-Lail, N. I., \& Camesano, T. A. (2003). Polysaccharide properties probed with atomic force microscopy. Journal of Microscopy, 212, 217-238.

Ansboro, S., Hayes, J. S., Barron, V., Browne, S., Howard, L., Greiser, U., et al. (2014).

Chondromimetic microsphere for in situ spatially controlled chondrogenic differentiation of human mesenchymal stem cells. Journal of Controlled Release, 179, 42-51.

Atkins, E. D., \& Sheehan, J. K. (1971). The molecular structure of hyaluronic acid. Biochemical Journal, 125, 92P.

Bian, L., Zhai, D. Y., Tous, E., Rai, R., Mauck, R. L., \& Burdick, J. A. (2011). Enhanced MSC chondrogenesis following delivery of TGF- $\beta 3$ from alginate microspheres within hyaluronic acid hydrogels. In Vitro and In Vivo. Biomaterials, 32, 6425-6434.

Braccini, I., \& Pérez, S. (2001). Molecular basis of $\mathrm{Ca}^{2+}$-induced gelation in alginates and pectins: the egg-box model revisited. Biomacromolecules, 2, 1089-1096.

Bugamelli, F., Raggi, M. A., Orienti, I., \& Zecchi, V. (1998). Controlled insulin release from chitosan microparticles. Archiv der Pharmazie, 331, 133-138.

Casu, B., \& Lindahl, U. (2001). Structure and biological interactions of heparin and heparan sulfate. Advances in Carbohydrate Chemistry and Biochemistry, 57, 159-206.

Chen, W., Kim, J.-H., Zhang, D., Lee, K.-H., Cangelosi, G. A., Soelberg, S. D., et al. (2013). Microfluidic one-step synthesis of alginate microspheres immobilized with antibodies. Journal of the Royal Society Interface, 10, 20130566.

Coradin, T., Coupé, A., \& Livage, J. (2003). Interactions of bovine serum albumin and lysozyme with sodium silicate solutions. Colloids and Surfaces B Biointerfaces, 29, 189-196.

Cristini, V., \& Tan, Y. C. (2004). Theory and numerical simulation of droplet dynamics in complex flows-a review. Lab on a Chip, 4, 257-264. 
576

577

578

579

580

581

582

583

584

585

586

587

588

589

590

591

592

593

594

595

596

597

598

599

600

DeFail, A. J., Chu, C. R., Izzo, N., \& Marra, K. G. (2006). Controlled release of bioactive TGF-beta 1 from microspheres embedded within biodegradable hydrogels. Biomaterials, 27, 1579-1585.

Freeman, I., Kedem, A., \& Cohen, S. (2008). The effect of sulfation of alginate hydrogels on the specific binding and controlled release of heparin-binding proteins. Biomaterials, 29, 3260-3268.

Fundueanu, G., Nastruzzi, C., Carpov, A., Desbrieres, J., \& Rinaudo, M. (1999). Physico-chemical characterization of Ca-alginate microparticles produced with different methods. Biomaterials, 20, 1427-1435.

Garstecki, P., Fuerstman, M. J., Stone, H. A., \& Whitesides, G. M. (2006). Formation of droplets and bubbles in a microfluidic T-junction—scaling and mechanism of break-up. Lab on a Chip, 6, 437-446.

Genes, N. G., Rowley, J. A., Mooney, D. J., \& Bonassar, L. J. (2004). Effect of substrate mechanics on chondrocyte adhesion to modified alginate surfaces. Archives of Biochemistry and Biophysics, 422, 161-167.

Gérard, C., Bordeleau, L.-J., Barralet, J., \& Doillon, C. J. (2010). The stimulation of angiogenesis and collagen deposition by copper. Biomaterials, 31, 824-831.

Grant, G. T., Morris, E. R., Rees, D. A., Smith, P. J. C., \& Thom, D. (1973). Biological interactions between polysaccharides and divalent cations: the egg-box model. FEBS Letter, 32, 195-198.

Gunning, A. P., Kirby, A. R., Morris, V. J., Wells, B., \& Brooker, B. E. (1995). Imaging bacterial polysaccharides by AFM. Polymer Bulletin, 34, 615-619.

Hoppe, A., Güldal, N. S., \& Boccaccini, A. R. (2011). A review of the biological response to ionic dissolution products from bioactive glasses and glass-ceramics. Biomaterials, 32, 2757-2774.

Jay, S. M., \& Saltzman, W. M. (2009). Controlled delivery of VEGF via modulation of alginate microparticle ionic crosslinking. Journal of Controlled Release, 134, 26-34.

Kamerling, J. P., Gerwing, G. J., Vliegenthart, J. F., \& Clamp, J. R. (1975). Characterization by gasliquid chromatography-mass spectrometry and proton-magnetic-resonance spectroscopy of 
601 pertrimethylsilyl methyl glycosides obtained in the methanolysis of glycoproteins and 602 glycopeptides. Biochemical Journal, 151, 491-495.

603 Kim, B. S., Oh, J. M., Kim, K. S., Seo, K. S., Cho, J. S., Khang, G., et al. (2009). BSA-FITC-loaded 604 microcapsules for in vivo delivery. Biomaterials, 30, 902-909.

605 Koppolu, B., Smith, S. G., Ravindranathan, S., Jayanthi, S., Kumar, T. K. S., \& Zaharoff, D. A. 606 (2014). Controlling chitosan-based encapsulation for protein and vaccine delivery. Biomaterials, 35 , $607 \quad 4382-4389$.

608 Lee, K. Y., \& Mooney, D. J. (2001). Hydrogels for tissue engineering. Chemical Reviews, 101, $609 \quad 1869-1879$.

610 Lim, J. J., Hammoudi, T. M., Bratt-Leal, A. M., Hamilton, S. K., Kepple, K. L., Bloodworth, N. C., 611 et al. (2011). Development of nano- and microscale chondroitin sulfate particles for controlled 612 growth factor delivery. Acta Biomaterialia, 7, 986-995.

613 Marquis, M., Renard, D., \& Cathala, B. (2012). Microfluidic generation and selective degradation 614 of biopolymer-based Janus microbeads. Biomacromolecules, 13, 1197-1203.

615 Marquis, M., Davy, J., Fang, A., \& Renard, D. (2014). Microfluidics-assisted diffusion self616 assembly: toward the control of the shape and size of pectin hydrogel microparticles. 617 Biomacromolecules, 15, 1568-1578.

618 Marquis, M., Davy, J., Cathala, B., \& Renard, D. (2015). Microfluidics assisted generation of 619 innovative polysaccharide hydrogel microparticles. Carbohydrate Polymers, 116, 189-199.

620 Niu, X., Feng, Q., Wang, M., Guo, X., \& Zheng, Q. (2009). Porous nano-HA/collagen/PLLA 621 scaffold containing chitosan microspheres for controlled delivery of synthetic peptide derived from 622 BMP-2. Journal of Controlled Release, 134, 111-117.

623 Olsson, E., \& Kreiss, G. (2005). A conservative level set method for two phase flow. Journal of 624 Computational Physics, 210, 225-246. 
625 Ouwerx, C., Velings, N., Mestdagh, M. M., \& Axelos, M. A. V. (1998). Physico-chemical properties 626 and rheology of alginate gel beads formed with various divalent cations. Polymer Gels and $627 \quad$ Networks, 6, 393-408.

628 Park, J. S., Na, K., Woo, D. G., Yang, H. N., \& Park, K. H. (2009). Determination of dual delivery 629 for stem cell differentiation using dexamethasone and TGF- $\beta 3$ in/on polymeric microspheres. 630 Biomaterials, 30, 4796-4805.

631 Pessi, J., Santos, H. A., Miroshnyk, I., Yliruusi, J., Weitz, D. A., \& Mirza, S. (2014). Microfluidics632 assisted engineering of polymeric microcapsules with high encapsulation efficiency for protein drug 633 delivery. International Journal of Pharmaceutics, 472, 82-87.

634 Raguénès, G., Christen, R., Guezennec, J., Pignet, P., \& Barbier, G. (1997). Vibrio diabolicus sp. 635 nov., a new polysaccharide-secreting organism isolated from a deep-sea hydrothermal vent 636 polychaete annelid, Alvinella pompejana. International Journal of Systematic Bacteriology, 47, $637989-995$.

638 Rodríguez, J. P., Ríos, S., \& González, M. (2002). Modulation of the proliferation and 639 differentiation of human mesenchymal stem cells by copper. The Journal of Cellular Biochemistry, $640 \quad 85,92-100$.

641 Rougeaux, H., Kervarec, N., Pichon, R., \& Guezennec, J. (1999). Structure of the 642 exopolysaccharide of Vibrio diabolicus isolated from a deep-sea hydrothermal vent. Carbohydrate Research, 322, 40-45.

644 Sarrazin, F., Bonometti, T., Prat, L., Gourdon, C., \& Magnaudet, J. (2008). Hydrodynamic 645 structures of droplets engineered in rectangular micro-channels. Microfluidics and Nanofluidics, 5, $646 \quad 131-137$.

647 Senni, K., Gueniche, F., Yousfi, M., Fioretti, F., Godeau, G., Colliec-Jouault, S., et al. (2008). 648 Sulfated depolymerized derivatives of exopolysaccharides (Eps) from mesophilic marine bacteria, 649 method for preparing same, and uses thereof in tissue regeneration. US Patent 2008/0,131,472. 
650 Senni, K., Gueniche, F., Changotade, S., Septier, D., Sinquin, C., Ratiskol, J., et al. (2013). Unusual 651 glycosaminoglycans from a deep sea hydrothermal bacterium improve fibrillar collagen structuring 652 and fibroblast activities in engineered connective tissues. Marine Drugs, 11, 1351-1369.

653 Silva, C. M., Ribeiro, A. J., Ferreira, D., \& Veiga, F. (2006). Insulin encapsulation in reinforced 654 alginate microspheres prepared by internal gelation. European Journal of Pharmaceutical Sciences, $65529,148-159$.

656 Smidsrød,O., \& Skjåk-Bræk, G. (1990). Alginate as immobilization matrix for cells. Trends in 657 Biotechnology, 8, 71-78.

658 Suh, J.-K. F., \& Matthew, H. W. T. (2000). Application of chitosan-based polysaccharide 659 biomaterials in cartilage tissue engineering: a review. Biomaterials, 21, 2589-2598.

660 Thorsen, T., Roberts, R. W., Arnold, F. H., \& Quake, S. R. (2001). Dynamic pattern formation in a 661 vesicle-generating microfluidic device. Physical Review Letters, 86, 4163-4166.

662 Xia, Y. N., \& Whitesides, G. M. (1998). Soft Lithography. Annual Review of Materials Science, 28, $663 \quad 153-184$.

664 Xu, Q., Hashimoto, M., Dang, T. T., Hoare, T., Kohane, D. S., Whitesides, G. M., et al. (2009). 665 Preparation of monodisperse biodegradable polymer microparticles using a microfluidic flow666 focusing device for controlled drug delivery. Small, 13, 1575-1581.

667 Zanchetta, P., Lagarde, N., \& Guezennec, J. (2003). A new bone-healing material: a hyaluronic 668 acid-like bacterial exopolysaccharide. Calcified Tissue International, 72, 74-79.

669 Zcharia, E., Zilka, R., Yaar, A., Yacoby-Zeevi, O., Zetser, A., Metzger, S., et al. (2005). Heparanase 670 accelerates wound angiogenesis and wound healing in mouse and rat models. The FASEB Journal, $671 \quad 19,211-221$.

672 Zhang, H., Tumarkin, E., Sullan, R. M. A., Walker, G. C., \& Kumacheva, E. (2007). Exploring 673 microfluidic routes to microgels of biological polymers. Macromolecular Rapid Communications, $674 \quad 28,527-538$. 
675 Zykwinska, A., Gaillard, C., Boiffard, M.-H., Thibault, J.-F., \& Bonnin, E. (2009). "Green labelled"

676 pectins with gelling and emulsifying properties can be extracted by enzymatic way from 677 unexploited sources. Food Hydrocolloid, 23, 2468-2477.

678 